\title{
Regulation of major histocompatibility complex class II genes
}

\author{
Nancy M. Choi ${ }^{1}$, Parimal Majumder ${ }^{1}$, and Jeremy M. Boss ${ }^{2}$ \\ Department of Microbiology \& Immunology, Emory University School of Medicine, 1510 Clifton \\ Road, Atlanta, GA 30322, Telephone: 404-727-5973
}

Nancy M. Choi: nmchoi@emory.edu; Parimal Majumder: pmajumd@emory.edu

\section{Summary}

\begin{abstract}
The major histocompatibility complex class II (MHC-II) genes are regulated at the level of transcription. Recent studies have shown that chromatin modification is critical for efficient transcription of these genes, and a number of chromatin modifying complexes recruited to MHC-II genes have been described. The MHC-II genes are segregated from each other by a series of chromatin elements, termed MHC-II insulators. Interactions between MHC-insulators and the promoters of MHC-II genes are mediated by the insulator factor CCCTC-binding protein and are critical for efficient expression. This regulatory mechanism provides a novel view of how the entire MHC-II locus is assembled architecturally and can be coordinately controlled.
\end{abstract}

\section{Introduction}

The major histocompatibility complex class II (MHC-II) genes function to present antigenic peptides to CD4 T cells. As a result of this action adaptive immune responses are initiated, maintained, and regulated. There are three isotypes of classical MHC-II genes in human (HLA-DR, DP, DQ), which are each formed of two polypeptides, $\alpha$ and $\beta$ chains (A and $\mathrm{B}$ genes) (Figure 1). The MHC-II-linked antigen presentation accessory genes (HLA-DM and $H L A-D O)$, as well as the unlinked invariant chain (Ii) gene, which encode proteins that function in translocation and peptide selection/loading of MHC-II molecules, are coordinately regulated with the classical MHC-II genes. MHC-II genes are constitutively expressed by professional antigen presenting cells (macrophages, dendritic cells, and B cells) and in thymic epithelial cells. These genes can be induced in non-immune cells (e.g., fibroblasts, epithelial cells, and endothelial cells, etc.) following exposure to cytokines, of which interferon- $\gamma($ IFN- $\gamma)$ is the most prominent [1]. One molecular genetic regulatory process controls the expression of this system and therefore the ability of cells to present antigens. This review will focus on advancements of the past decade that have determined the complex interplay between the promoter proximal cis- and trans-regulatory elements and the chromatin machinery that serves to activate gene expression, and the newly described chromatin architecture and dynamic organization of the locus.

\footnotetext{
(C) 2010 Elsevier Ltd. All rights reserved

${ }^{2}$ Corresponding author: Jeremy M. Boss jmboss@emory.edu.

$1_{\text {Co-first authors }}$
}

Publisher's Disclaimer: This is a PDF file of an unedited manuscript that has been accepted for publication. As a service to our customers we are providing this early version of the manuscript. The manuscript will undergo copyediting, typesetting, and review of the resulting proof before it is published in its final citable form. Please note that during the production process errors may be discovered which could affect the content, and all legal disclaimers that apply to the journal pertain.

Conflicts of Interest

The authors have no financial conflict of interest with regard to this work. 


\section{MHC-II promoter proximal elements and factors}

Until recently, control of this system was thought to occur primarily through the action of a highly conserved, promoter proximal, combinatorial cis-regulatory sequence located approximately 100-200 bp upstream of the transcription start site of each MHC-II gene. The sequence consists of the W/S/Z, X1, X2, and Y box elements [2]. NF-Y (nuclear factor-Y) complex, comprised of NF-Ya, NF-Yb, and NF-Yc, binds to the Y box. Due to the histone fold like structure of NF-Yb and NF-Yc [3], the NF-Y complex is thought to distort and compact the structure of DNA, thereby providing an additional level of specificity for combinatorial transcription factor binding [4,5]. NF-Y regulates many genes as the $\mathrm{Y}$ box contains the CCAAT sequence described many years ago as a canonical regulatory element. The $\mathrm{X} 2$ box is bound by CREB (cAMP response element binding protein) [6], and as its name implies, cAMP-dependent activation pathways modulate CREB's activity in other gene systems. However, although CREB and its phosphorylated form were found at the HLA-DRA X2 box, CREB phosphorylation was not essential and only mildly enhanced class II transcription [7]. CREB's role as a stabilizing partner of the $\mathrm{X} 1$ box proteins at the promoter is likely a more important function and a mechanism unique to the MHC-II system [7].

Studies of bare lymphocyte syndrome (BLS) patient-derived cell lines, which are defective for MHC-II gene expression, identified four complementation groups that were essential for MHC-II gene expression. The genetic bases for three of these were due to mutations in transcription factors that formed the X1 box binding factor RFX: RFX5 (regulatory factor X5), RFXAP, and RFX-B/RFXANK [2]. Solution structure studies of RFX protein domains suggest that the RFX complex likely exists as a heterotetramer with two RFX5 molecules and monomers of RFXAP and RFX-B [8,9]. Irrespective of MHC-II gene expression, RFX interacts directly with CREB and NF-Y, forming a combinatorial DNA-protein complex on the $\mathrm{X}-\mathrm{Y}$ box region. By itself this ternary complex is transcriptionally inactive, but is specifically recognized by CIITA (class II transactivator), which is required to activate transcription. CIITA mutations represent the genetic lesion of the fourth BLS complementation group, and of the above factors, is the only one that is rate limiting and highly regulated. As such, the presence/absence of CIITA determines if a cell can express MHC-II genes. Thus, CIITA is the master regulator of this system.

Despite being discovered more than 20 years ago, the role of the $\mathrm{W}$ box in this system is still not clear. Early reports suggested that RFX also bound to the W box [10]. In contrast, more recent data suggest that this is not the case as purified RFX complexes did not interact with the $\mathrm{W}$ box sequences [11] and mutations in the $\mathrm{W}$ box did not affect binding of RFX to the $\mathrm{X} 1$ box [12]. Instead, $\mathrm{W}$ box mutations led to decreases in CIITA binding [12]. The distance between the $\mathrm{W}$ and $\mathrm{X}$ box elements was also crucial for CIITA binding, which suggests the possibility of a yet unidentified W-box binding factor at this locus [12].

CIITA is expressed from three major promoters in a cell type and cytokine dependent manner. Each promoter produces a slightly different $\mathrm{N}$-terminal isoform, but the need if any for distinct isoforms is still not clear. The domain features of CIITA were used to define and discover the NLR/NOD/NACHT family of molecules that play multiple roles in innate immune responses [13,14]. CIITA was also shown to upregulate human MHC-I genes in response to IFN- $\gamma$, although the immunological relevance of this function was not fully established. Most recently, the NLR family member NLRC5 (nucleotide binding domain and leucine rich repeat containing 5) was shown to be the likely factor that is responsible for MHC-I induction by IFN- $\gamma$ in vivo [15]. NLRC5 does not regulate MHC-II genes. Thus, NLR family members regulate both MHC-I and MHC-II genes. It will be important to 
ultimately determine how these transcriptional activators are able to function with specificity on the different MHC genes.

Posttranslational modifications of CIITA are important to modulate its function [16]. CIITA phosphorylation is involved in regulating CIITA nuclear localization and self-interaction. Acetylation of CIITA by acetyltransferases PCAF (p300/CBP associated factor) and CBP (CREB binding protein) may be important for its nuclear localization and activity [16]. By contrast, class I histone deacetylase HDAC1 (histone deacetylase 1) had a negative regulatory role by interacting with CIITA to restrict its interaction with the enhanceosome complex [17], and HDAC2 was shown to directly deacetylate CIITA and target it towards proteasomal degradation [16]. Monoubiquitylation of CIITA stabilizes the protein at the MHC-II promoters, and phosphorylation of a nearby serine residue was required for this modification, which suggests an intricate crosstalk between posttranslational modifications that are necessary for optimal activity of CIITA [18].

\section{MHC-II epigenetic regulation through histones}

Modification or the remodeling of nucleosomes has been shown to be associated with gene expression and silencing $[19,20]$. The HLA-DRA gene promoter was one of the first immune system genes in which this connection was demonstrated [21]. At least four multisubunit complexes are recruited to MHC-II promoters to mediate these effects: CBP/p300, STAGA/ ATAC (SPT3-TAF9-GCN5/PCAF/Ada Two-A-containing), COMPASS/MLL (complex associated with Set1/mixed lineage leukemia), and SWI/SNF (mating type switching/ sucrose non-fermenting).

Histone acetylation is important for MHC-II gene expression. Following IFN- $\gamma$ treatment histone $\mathrm{H} 4$ acetylation occurred in parallel with RNA polymerase II recruitment preceding transcriptional initiation, while histone $\mathrm{H} 3$ acetylation levels increased with productive mRNA transcription and were dependent on RNA pol II elongation [22]. CBP/p300, PCAF, and GCN5 (general control nonderepressible 5) are histone acetyltransferases (HAT) that are recruited by CIITA to MHC-II promoters [23-25]. Of these HATs, GCN5 and PCAF function as components of STAGA and ATAC complexes, and with $\mathrm{CBP} / \mathrm{p} 300$ are likely responsible for all of the activation associated acetylation marks at the MHC-II promoters [26]. CIITA itself has been shown to possess HAT activity as well [27]. The ATPase Sug1, a component of the $19 \mathrm{~S}$ proteasome complex present in the nucleus, associates with acetylated histone $\mathrm{H} 3$ and appears to be required for increased acetylation or stability of this mark at MHC-II promoters. Intriguingly, Sug1 also associates with CBP and this association is important for the recruitment of CBP to MHC-II genes [28]. Histone hyperacetylation through the inhibition of HDACs induced recruitment of enhanceosome components, but also led to the activation of MHC-II gene expression in the absence of CIITA. This suggests that there are multiple roles of histone acetylation at the MHC-II promoters: at least one modifying CIITA and another modifying the chromatin structure such that transcription can be initiated more efficiently [17,29].

Several histone methylation marks associated with gene activation are also found at MHC-II promoters. The COMPASS/MLL type complexes are responsible for histone H3 lysine 4 methylation (H3K4me) from yeast to humans and also function at MHC-II genes. These complexes have a core histone methyltransferase (HMT) component accompanied by WDR5 (WD repeat domain 5), ASH2L (ash2-like), and RBBP5 (retinoblastoma binding protein 5) [30], which were bound at the $H L A-D R A$ promoter [31]. BRE1 (brefeldin A sensitivity 1) and UTX1 (ubiquitously transcribed tetratricopeptide repeat gene on X chromosome 1), which are also involved in COMPASS/MLL related activation of genes, were also recruited to the MHC-II promoters with IFN- $\gamma$ treatment $[18,32]$. Coupled with 
these events following IFN- $\gamma$ treatment, was the induced interaction between MLL and PML (promyelocytic leukemia) and relocation of the MHC-II promoters to PML nuclear bodies, which was important for sustaining H3K4me2 at the MHC-II promoters [33]. H3K4me2 histone modification is associated with transcriptional competency of a region and together with relocation to PML bodies, suggests a mechanism of transcriptional memory through chromosomal restructuring that may have a lasting effect on gene expression after the stimulus is removed.

Remodeling of MHC-II promoter nucleosomes by the SWI/SNF complex [34] and nucleosome eviction at the $\mathrm{W}-\mathrm{X}-\mathrm{Y}$ box was also important for proper expression and transcription start site selection of MHC-II genes [35]. The mechanism of establishing the nucleosome free promoter region was unique as it was not mediated by strong nucleosome positioning signals but through binding of the $\mathrm{W}-\mathrm{X}-\mathrm{Y}$ box factors [35].

With all of these complexes and perhaps other factors functioning to modify the chromatin structure and activate transcription, two important issues can be raised. The first question is whether any of these events are unique to MHC-II genes. Because the chromatin remodeling complexes share components with one another, it is likely that some complex components or their isoforms are specific to this system while others are ubiquitous to all genes. Even if these are ubiquitous events, the second issue is how are these activities coordinated in both time and space at MHC-II promoters. CIITA is clearly the pivotal factor that facilitates the chromatin modification machinery and the activation of the system. As described below, these events are not sufficient to fully express these genes.

\section{Long-range chromosomal regulation of MHC-II}

In the late 1980s a distal X-Y box element was identified in the murine $I-E a$ locus that was associated with its correct tissue specific regulation when introduced as a transgene [36]. In the HLA-DRA system, a functional distal X-Y element was also found [37]. A screen for additional $\mathrm{X}$-Y motifs found 32 non-promoter $\mathrm{X}$ box-like (XL) sequences within the MHCII locus. Like the above sequences, some were functional, having properties similar to the promoter proximal W-X-Y boxes [38]. In contrast to the above elements, the $X L 9$ site, located in the intergenic region between HLA-DRBI and -DQA1, did not bind RFX or CIITA, had no enhancer activity, but was in chromatin that was highly acetylated [39]. Because $X L 9$ was between the $H L A-D R$ and $-D Q$ subregions, there was the possibility that the region functioned as a chromatin boundary/insulator element.

Chromatin boundary/insulator elements function to organize chromatin into independent regulatory domains [40]. Insulators have the property of being able to block the activity of an enhancer from activating a promoter when placed between them. Some insulators also serve as barriers or boundary elements by preventing the encroachment of heterochromatin into active genes [41]. These activities have been ascribed to CTCF (CCCTC binding factor), although it is likely that CTCF interacts with several other factors to mediate these functions [42]. CTCF, a zinc finger DNA-binding protein that can homodimerize, is associated with the formation of long range chromatin loops between adjacent CTCF sites. Depending on the cell type and genetic context, and likely through the chromatin loops that it organizes, CTCF is ascribed transcriptional repression or activation functions [43-45]. Because its binding to DNA is methylation dependent, CTCF has been shown to play a major role in genomic imprinting [45] and X-chromosome inactivation in mammals [46]. But precisely how these regulatory events are mediated is not known.

Indeed, CTCF was found to bind to sequences close to $X L 9$, and this region mediated strong enhancer blocking activity [47]. For simplicity, the initial name of the region was retained. Further analyses showed that CTCF interacted with CIITA and was required for a 
chromatin-looping event between the $X L 9$ and proximal promoter regions of $H L A-D R B 1$ and $H L A-D Q A 1$ genes [47]. CTCF depletion by RNAi resulted in a substantial reduction in HLA-DRB1 and HLA-DQA1 gene expression. These data suggested that CTCF and potentially $X L 9$ were critical for expression of these and perhaps all MHC-II genes.

The question of whether CTCF was required for all MHC-II genes was addressed by using RNAi depletion. All CIITA-regulated genes within the MHC-II locus required CTCF for maximal expression [48]. The five non-CIITA genes within the locus (TAP1, TAP2, PSMB8, $P S M B$ 9, and BRD2) were not affected by CTCF depletion. The question as to whether XL9 was the only CTCF site that interacted with MHC-II gene promoters was also addressed. Genome wide studies identified 17 CTCF sites within the human MHC-II, including XL9 [49,50]; however, traditional chromatin immunoprecipitation studies showed that only ten of those sites truly bound CTCF in B cells and several other cell types (Figure 1) [48]. Intriguingly, the validated sites resided between MHC-II subregions, such that they surrounded the subregions and for the most part isolated the CIITA-regulated genes from the others (Figure 1). For example, $C l$ and $X L 9$ surround the $H L A-D R$ subregion, whereas $X L 9$ and $C 2$ surround $H L A-D Q$. This may suggest that part of the duplication process that created multiple MHC-II genes may have required a CTCF site to be present during the event as it is part of the regulatory machinery.

Using chromatin conformation capture (3C) assays, procedures that allow the detection of long-range chromatin interactions [51], two sets of interactions involving these CTCF sites were uncovered [48]. A basal state of interactions between each of the CTCF sites occurred irrespective of MHC-II gene expression and was similar in B cells and fibroblasts (Figure 3). For example, $C l$ was found to interact with its closest neighbor $X L 9$ and to a lesser degree with $C 2$, which is $250 \mathrm{~kb}$ away. Similarly $X L 9$ could also interact with $C 2$. The interactions appear to decrease substantially as the distances exceed $200 \mathrm{~kb}$. These chromatin loops have the potential therefore to isolate the $H L A-D R$ and $H L A-D Q$ subregions from each other and from the non-CIITA regulated genes. As such we have termed these sites "MHC-II insulators." While the data do not distinguish between single and multiple interactions, the fact that CTCF can form homodimers suggests that only a single set of basal interactions for MHC-II insulators exists for any chromosome. However, each chromosome within a cell may have a different set of interactions. This latter concept would suggest that a population of MHC-II expressing cells would have multiple MHC-II chromatin structures.

The second set of interactions overlays the first and occurs in the transcriptionally active state (Figure 3). These interactions are dependent on CIITA binding at MHC-II promoters and likely include the aid of all of the factors described in the first part of this review. In this set, CIITA bound W-X-Y box DNA regions interact directly with CTCF bound MHC-II insulators. For example, CTCF bound at the $C l$ site interacts with the HLA-DRA proximal promoter forming a second long-range chromatin loop (Figure 3). Depletion of CTCF by RNAi disrupts MHC-II insulator interactions and transcription of MHC-II genes. Each CIITA bound proximal promoter region can interact with different CTCF bound MHC-II insulator regions. Again, the data do not distinguish between promoters interacting with one or more MHC-II insulators simultaneously. However, to do so would likely require that multiple binding sites are available for each interaction. Because these interactions are CIITA dependent, they are induced by IFN- $\gamma$ exposure of cells, which induces the expression of CIITA. Aside from overall distance, it is not clear at this point if there are preferred combinations for MHC-II promoter regions and MHC-II insulators. While the overall functions of the MHC-II insulators appear to be similar, it is not known if some MHC-II insulators are more efficient at driving MHC-II transcription than others. If this were the case, then some MHC-II insulator-promoter interactions could result in different levels of mRNA transcribed. 
Recently, cohesins have been found to be associated with $75 \%$ of genome wide CTCF sites in humans [52-54]. Cohesin is a multisubunit-ringed complex that was initially thought to only function in the pairing of sister chromatids during cell division. With the finding of cohesin subunits in all stages of the cell cycle, and its association with CTCF, it has now been shown that cohesin is necessary for the enhancer blocking activity of CTCF [53]. Thus, it was not surprising to find that cohesin was associated with each of the MHC-II insulators. It will be interesting to determine if cohesin is also required for MHC-II transcription and/or to form the architecture that is now attributed to CTCF binding at the MHC-II insulator regions. In considering the ringed structure of cohesin, it is tempting to speculate that it will provide stability to either or both sets of interactions observed in this system.

These newly described sets of interactions and dependence on CTCF for MHC-II transcription was not an expected mechanism. This raises the important question of how these structures contribute to the regulation of this system. Because CTCF bound sequences are in highly acetylated chromatin, MHC-II insulators organize the chromatin into a steadystate architecture that is readily accessible to transcriptional regulatory machinery. This is consistent with the fact that active histone marks were reduced following CTCF depletion [47]. Alternatively, the CTCF-related interactions may stabilize the association of the histone modifying complexes. Another possibility is that the MHC-II insulators may cause the formation of a transcriptional hub/factory, a location that might be associated with high concentrations of transcription components and machinery [55]. The presence of MHC-II gene promoters in transcription factories would also have the advantage of allowing these genes to be expressed efficiently.

\section{Conclusion}

The proper regulation of MHC-II genes is fundamental to achieving adaptive immunity. The system is complex with numerous pathways that not only target the modification of the transcription factors specific to the system but also the chromatin modification machinery that is required for expression. Once elucidated the components and processes in these pathways represent targets to manipulate the expression of this system in a therapeutic setting. The newly introduced role of chromatin insulators and the potential dynamic movement of MHC-II loci to structures within the nucleus also provide new insights into how genes are regulated and what the necessary steps are to coordinate the expression of a multi-gene family and biological mechanism such as antigen presentation.

\section{Acknowledgments}

We thank members of the lab for their suggestions and comments. This work was supported by National Institutes of Health grants AI34000 and GM47310

\section{Reference}

1. Collins T, Korman AJ, Wake CT, Boss JM, Kappes DJ, Fiers W, Ault KA, Gimbrone MA Jr, Strominger JL, Pober JS. Immune interferon activates multiple class II major histocompatibility complex genes and the associated invariant chain gene in human endothelial cells and dermal fibroblasts. Proceedings of the National Academy of Sciences of the United States of America 1984;81:4917-4921. [PubMed: 6431411]

2. Reith W, Mach B. The bare lymphocyte syndrome and the regulation of MHC expression. Annu Rev Immunol 2001;19:331-373. [PubMed: 11244040]

3. Baxevanis AD, Arents G, Moudrianakis EN, Landsman D. A variety of DNA-binding and multimeric proteins contain the histone fold motif. Nucleic Acids Research 1995;23:2685-2691. [PubMed: 7651829] 
4. Ronchi A, Bellorini M, Mongelli N, Mantovani R. CCAAT-box binding protein NF-Y (CBF, CP1) recognizes the minor groove and distorts DNA. Nucleic Acids Research 1995;23:4565-4572. [PubMed: 8524643]

5. Guerra RF, Imperadori L, Mantovani R, Dunlap DD, Finzi L. DNA compaction by the nuclear factor-Y. Biophys J 2007;93:176-182. [PubMed: 17434933]

6. Moreno CS, Beresford GW, Louis-Plence P, Morris AC, Boss JM. CREB regulates MHC class II expression in a CIITA-dependent manner. Immunity 1999;10:143-151. [PubMed: 10072067]

7. Lochamy J, Rogers EM, Boss JM. CREB and phospho-CREB interact with RFX5 and CIITA to regulate MHC class II genes. Mol Immunol 2007;44:837-847. [PubMed: 16730065]

8. Garvie CW, Stagno JR, Reid S, Singh A, Harrington E, Boss JM. Characterization of the RFX complex and the RFX5(L66A) mutant: implications for the regulation of MHC class II gene expression. Biochemistry 2007;46:1597-1611. [PubMed: 17279624]

9. Laird KM, Briggs LL, Boss JM, Summers MF, Garvie CW. Solution Structure of the Heterotrimeric Complex between the Interaction Domains of RFX5 and RFXAP from the RFX Gene Regulatory Complex. J Mol Biol. 2010 RFX5 interacting domains are shown to form antiparallel coliled-coils that interact with RFXAP. RFXAP in turn has a hydrophobic pocket that is predicted to serve as the binding site for RFX-B.

10. Jabrane-Ferrat N, Fontes JD, Boss JM, Peterlin BM. Complex architecture of major histocompatibility complex class II promoters: reiterated motifs and conserved protein-protein interactions. Mol Cell Biol 1996;16:4683-4690. [PubMed: 8756625]

11. Garvie CW, Boss JM. Assembly of the RFX complex on the MHCII promoter: role of RFXAP and RFXB in relieving autoinhibition of RFX5. Biochim Biophys Acta 2008;1779:797-804. [PubMed: 18723135]

12. Muhlethaler-Mottet A, Krawczyk M, Masternak K, Spilianakis C, Kretsovali A, Papamatheakis J, Reith W. The S box of major histocompatibility complex class II promoters is a key determinant for recruitment of the transcriptional co-activator CIITA. J Biol Chem 2004;279:40529-40535. [PubMed: 15271997]

13. Ting JP, Lovering RC, Alnemri ES, Bertin J, Boss JM, Davis BK, Flavell RA, Girardin SE, Godzik A, Harton JA, et al. The NLR gene family: a standard nomenclature. Immunity 2008;28:285-287. [PubMed: 18341998]

14. Harton JA, Linhoff MW, Zhang J, Ting JP. Cutting edge: CATERPILLER: a large family of mammalian genes containing CARD, pyrin, nucleotide-binding, and leucine-rich repeat domains. J Immunol 2002;169:4088-4093. [PubMed: 12370334]

15. Meissner TB, Li A, Biswas A, Lee KH, Liu YJ, Bayir E, Iliopoulos D, van den Elsen PJ, Kobayashi KS. NLR family member NLRC5 is a transcriptional regulator of MHC class I genes. Proc Natl Acad Sci U S A 2010;107:13794-13799. [PubMed: 20639463] NLRC5 is shown here to be induced by IFN $\gamma$ and regulate MHC-I genes.

16. Wu X, Kong X, Luchsinger L, Smith BD, Xu Y. Regulating the activity of class II transactivator by posttranslational modifications: exploring the possibilities. Molecular and Cellular Biology 2009;29:5639-5644. [PubMed: 19720744]

17. Zika E, Greer SF, Zhu X-S, Ting JP-Y. Histone deacetylase 1/mSin3A disrupts gamma interferoninduced CIITA function and major histocompatibility complex class II enhanceosome formation. Molecular and Cellular Biology 2003;23:3091-3102. [PubMed: 12697811]

18. Bhat KP, Truax AD, Greer SF. Phosporylation and Ubiquitination of degron proximal residues is essential for CIITA transactivation and MHC class II expression. Journal of Biological Chemistry 2010:1-18.

19. Clapier CR, Cairns BR. The biology of chromatin remodeling complexes. Annu Rev Biochem 2009;78:273-304. [PubMed: 19355820]

20. Jenuwein T, Allis CD. Translating the histone code. Science 2001;293:1074-1080. [PubMed: 11498575]

21. Beresford GW, Boss JM. CIITA coordinates multiple histone acetylation modifications at the HLA-DRA promoter. Nat Immunol 2001;2:652-657. [PubMed: 11429551] 
22. Rybtsova N, Leimgruber E, Seguin-Estévez Q, Dunand-Sauthier I, Krawczyk M, Reith W. Transcription-coupled deposition of histone modifications during MHC class II gene activation. Nucleic Acids Research 2007;35:3431-3441. [PubMed: 17478518]

23. Kretsovali A, Agalioti T, Spilianakis C, Tzortzakaki E, Merika M, Papamatheakis J. Involvement of CREB binding protein in expression of major histocompatibility complex class II genes via interaction with the class II transactivator. Mol Cell Biol 1998;18:6777-6783. [PubMed: 9774691]

24. Fontes JD, Kanazawa S, Jean D, Peterlin BM. Interactions between the class II transactivator and CREB binding protein increase transcription of major histocompatibility complex class II genes. Mol Cell Biol 1999;19:941-947. [PubMed: 9858618]

25. Spilianakis C, Papamatheakis J, Kretsovali A. Acetylation by PCAF enhances CIITA nuclear accumulation and transactivation of major histocompatibility complex class II genes. Molecular and Cellular Biology 2000;20:8489-8498. [PubMed: 11046145]

26. Nagy Z, Tora L. Distinct GCN5/PCAF-containing complexes function as co-activators and are involved in transcription factor and global histone acetylation. Oncogene 2007;26:5341-5357. [PubMed: 17694077]

27. Raval A, Howcroft TK, Weissman JD, Kirshner S, Zhu XS, Yokoyama K, Ting J, Singer DS. Transcriptional coactivator, CIITA, is an acetyltransferase that bypasses a promoter requirement for TAF(II)250. Mol Cell 2001;7:105-115. [PubMed: 11172716]

28. Koues OI, Dudley RK, Truax AD, Gerhardt D, Bhat KP, Mcneal S, Greer SF. Regulation of Acetylation at the Major Histocompatibility Complex Class II Proximal Promoter by the $19 \mathrm{~S}$ Proteasomal ATPase Sug1. Molecular and Cellular Biology 2008;28:5837-5850. [PubMed: 18662994] Authors demonstrate that 19S ATPase Sug1 is important for proper MHC-II expression by regulating acetylation of histones at the MHC-II promoter through recruitment of CBP.

29. Gialitakis M, Kretsovali A, Spilianakis C, Kravariti L, Mages J, Hoffmann R, Hatzopoulos AK, Papamatheakis J. Coordinated changes of histone modifications and HDAC mobilization regulate the induction of MHC class II genes by Trichostatin A. Nucleic Acids Research 2006;34:765-772. [PubMed: 16452299]

30. Eissenberg JC, Shilatifard A. Histone H3 lysine 4 (H3K4) methylation in development and differentiation. Developmental Biology 2009:1-10.

31. Koues OI, Mehta NT, Truax AD, Dudley RK, Brooks JK, Greer SF. Roles for common MLL/ COMPASS subunits and the 19S proteasome in regulating CIITA pIV and MHC class II gene expression and promoter methylation. 2010:1-11.

32. Koues OI, Dudley RK, Mehta NT, Greer SF. The 19S proteasome positively regulates histone methylation at cytokine inducible genes. Biochim Biophys Acta 2009;1789:691-701. [PubMed: 19660582]

33. Gialitakis M, Arampatzi P, Makatounakis T, Papamatheakis J. Gamma interferon-dependent transcriptional memory via relocalization of a gene locus to PML nuclear bodies. Molecular and Cellular Biology 2010;30:2046-2056. [PubMed: 20123968] Following interferon- $\gamma$ treatment, an accessible chromatin environment was sustained by $\mathrm{H} 3 \mathrm{~K} 4 \mathrm{me} 2$ modifciation of the HLA-DRA gene, and these primed cells had a hightened response to restimulation. The HLA-DRA locus relocalized to PML-nuclear bodies, and this event was mediated through an interaction between the MLL complex and PML.

34. Mudhasani R, Fontes JD. Multiple interactions between BRG1 and MHC class II promoter binding proteins. Mol Immunol 2005;42:673-682. [PubMed: 15781111]

35. Leimgruber E, Seguin-Estevez Q, Dunand-Sauthier I, Rybtsova N, Schmid CD, Ambrosini G, Bucher P, Reith W. Nucleosome eviction from MHC class II promoters controls positioning of the transcription start site. Nucleic Acids Research 2009;37:2514-2528. [PubMed: 19264803] This paper describes the unique mechanism of how the proximal promoter region of MHC-II genes maintain a nucleosome free region and its importance in selecting the proper transcriptional start site.

36. van Ewijk W, Ron Y, Monaco J, Kappler J, Marrack P, Le Meur M, Gerlinger P, Durand B, Benoist C, Mathis D. Compartmentalization of MHC class II gene expression in transgenic mice. Cell 1988;53:357-370. [PubMed: 3259162] 
37. Masternak K, Peyraud N, Krawczyk M, Barras E, Reith W. Chromatin remodeling and extragenic transcription at the MHC class II locus control region. Nat Immunol 2003;4:132-137. [PubMed: 12524537]

38. Gomez JA, Majumder P, Nagarajan UM, Boss JM. X box-like sequences in the MHC class II region maintain regulatory function. J Immunol 2005;175:1030-1040. [PubMed: 16002703]

39. Majumder P, Gomez JA, Boss JM. The human major histocompatibility complex class II HLADRB1 and HLA-DQA1 genes are separated by a CTCF-binding enhancer-blocking element. J Biol Chem 2006;281:18435-18443. [PubMed: 16675454]

40. West AG, Fraser P. Remote control of gene transcription. Hum Mol Genet 2005;14(Spec No 1):R101-R111. [PubMed: 15809261]

41. Gaszner M, Felsenfeld G. Insulators: exploiting transcriptional and epigenetic mechanisms. Nat Rev Genet 2006;7:703-713. [PubMed: 16909129]

42. Bell AC, West AG, Felsenfeld G. The Protein CTCF Is Required for the Enhancer Blocking Activity of Vertebrate Insulators. Cell 1999;98:387-396. [PubMed: 10458613]

43. Filippova GN, Fagerlie S, Klenova EM, Myers C, Dehner Y, Goodwin G, Neiman PE, Collins SJ, Lobanenkov VV. An exceptionally conserved transcriptional repressor, CTCF, employs different combinations of zinc fingers to bind diverged promoter sequences of avian and mammalian c-myc oncogenes. Mol Cell Biol 1996;16:2802-2813. [PubMed: 8649389]

44. Vostrov AA, Taheny MJ, Quitschke WW. A region to the N-terminal side of the CTCF zinc finger domain is essential for activating transcription from the amyloid precursor protein promoter. J Biol Chem 2002;277:1619-1627. [PubMed: 11706010]

45. Hark AT, Schoenherr CJ, Katz DJ, Ingram RS, Levorse JM, Tilghman SM. CTCF mediates methylation-sensitive enhancer-blocking activity at the H19/Igf2 locus. Nature 2000;405:486-489. [PubMed: 10839547]

46. Chao W, Huynh KD, Spencer RJ, Davidow LS, Lee JT. CTCF, a candidate transacting factor for X-inactivation choice. Science 2002;295:345-347. [PubMed: 11743158]

47. Majumder P, Gomez JA, Chadwick BP, Boss JM. The insulator factor CTCF controls MHC class II gene expression and is required for the formation of long-distance chromatin interactions. J Exp Med 2008;205:785-798. [PubMed: 18347100] Here, the CTCF insulator XL9 and the two flanking MHC-II genes, $H L A-D R B 1$ and $H L A-D Q A 1$, were shown to form chromatin loops in a transcription-dependent manner with IFN- $\gamma$ treatment. This looping event was mediated through protein-protein interactions between CTCF and the MHC-II enhanceosome transcription factors.

48. Majumder P, Boss JM. CTCF controls expression and chromatin architecture of the human major histocompatibility complex class II locus. Mol Cell Biol 2010;30:4211-4223. [PubMed: 20584980] CTCF was found to be required for maximal MHC-II gene transcription. The $10 \mathrm{CTCF}$ binding sites interacted with each other in a distance dependent manner, establishing a basal structure of the locus. Upon activation of the MHC-II gene expression, CIITA dependent interactions with the CTCF sites formed, creating a series of additional loops and importantly foci.

49. Kim TH, Abdullaev ZK, Smith AD, Ching KA, Loukinov DI, Green RD, Zhang MQ, Lobanenkov VV, Ren B. Analysis of the Vertebrate Insulator Protein CTCF-Binding Sites in the Human Genome. Cell 2007;128:1231-1245. [PubMed: 17382889]

50. Barski A, Cuddapah S, Cui K, Roh T-Y, Schones DE, Wang Z, Wei G, Chepelev I, Zhao K. HighResolution Profiling of Histone Methylations in the Human Genome. Cell 2007;129:823-837. [PubMed: 17512414]

51. Dekker J. The three 'C' s of chromosome conformation capture: controls, controls, controls. Nat Methods 2006;3:17-21. [PubMed: 16369547]

52. Stedman W, Kang H, Lin S, Kissil JL, Bartolomei MS, Lieberman PM. Cohesins localize with CTCF at the KSHV latency control region and at cellular c-myc and H19/Igf2 insulators. Embo J 2008;27:654-666. [PubMed: 18219272]

53. Wendt KS, Yoshida K, Itoh T, Bando M, Koch B, Schirghuber E, Tsutsumi S, Nagae G, Ishihara $\mathrm{K}$, Mishiro T, et al. Cohesin mediates transcriptional insulation by CCCTC-binding factor. Nature 2008;451:796-801. [PubMed: 18235444] 
54. Parelho V, Hadjur S, Spivakov M, Leleu M, Sauer S, Gregson HC, Jarmuz A, Canzonetta C, Webster Z, Nesterova T, et al. Cohesins functionally associate with CTCF on mammalian chromosome arms. Cell 2008;132:422-433. [PubMed: 18237772]

55. Sutherland H, Bickmore WA. Transcription factories: gene expression in unions? Nat Rev Genet 2009;10:457-466. [PubMed: 19506577] 


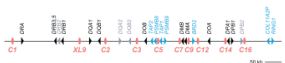

Figure 1. MHC-II locus

Schematic of classical and non-classical MHC-II genes (black), MHC-II pseudo-genes (grey), and non-MHC-II genes (blue) together form a very gene dense locus on the short arm of human chromosome 6 . The locus is punctuated with $10 \mathrm{CTCF}$ binding sites $(C 1-C 16$; pink) including XL9. 


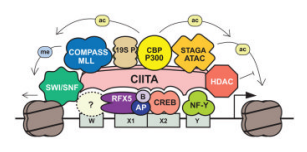

Figure 2. Promoter proximal MHC-II regulation

Highly conserved W-X-Y box is bound by RFX, CREB, and NF-Y. They form a unique structure that is recognized by CIITA. CIITA recruits indicated transcriptional coactivators and their associated complexes that modulate the activity of the enhanceosome proteins and modify nucleosomes (ac; acetylation, me; methylation) surrounding the nucleosome free region (NFR) to regulate transcription. $19 \mathrm{~S}$ P: $19 \mathrm{~S}$ proteasome regulatory complex. 
OFF state

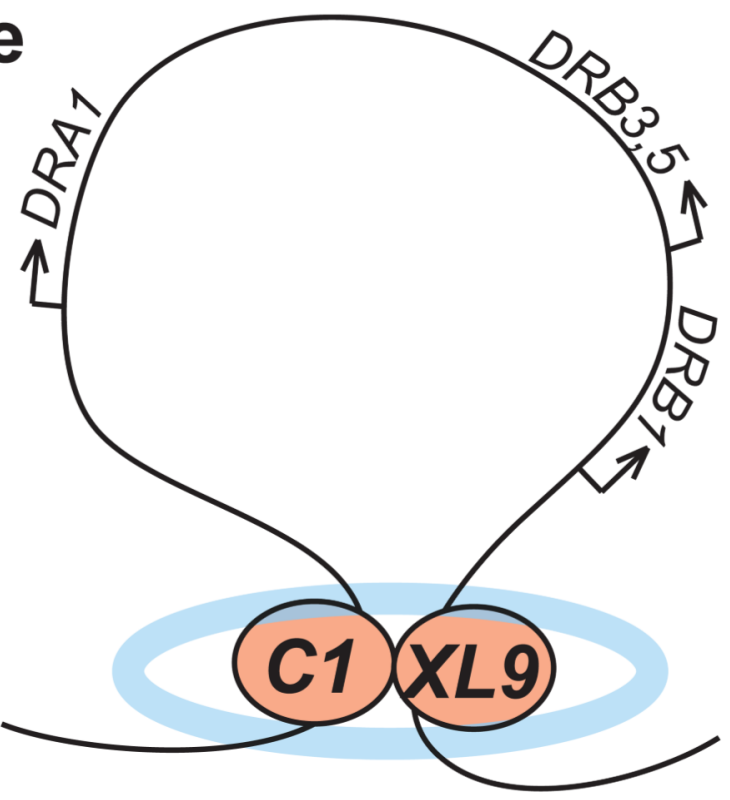

\section{ON state}

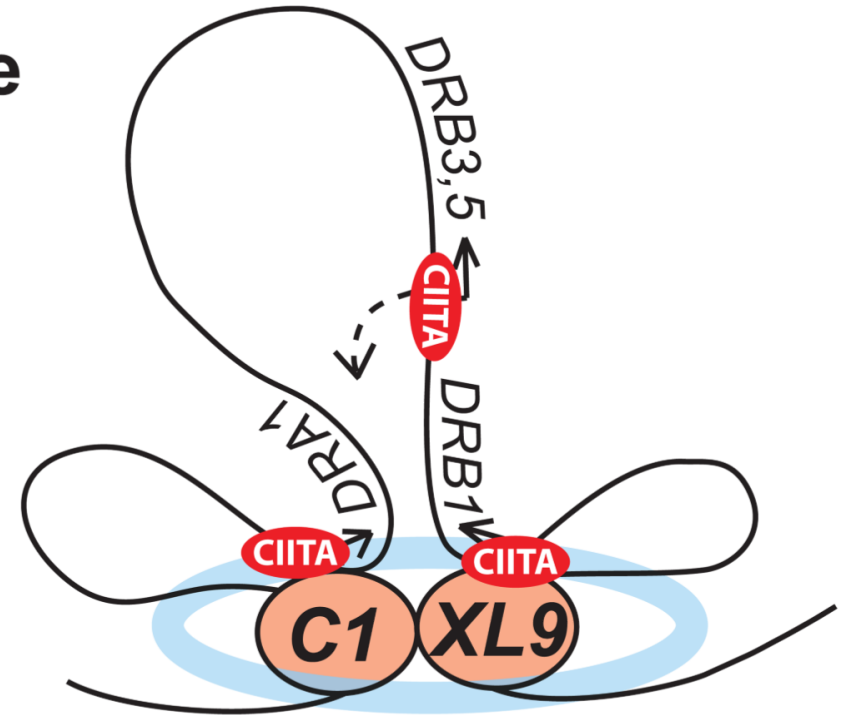

Figure 3. MHC-II insulator long-range looping model

In the 'OFF state', MHC-insulators bound by CTCF (orange), $C 1$ and XL9, interact to form a chromatin loop and interaction focus. Cohesin (blue) likely encircles the interacting CTCF foci maintaining the integrity/stability of the structure or its function. When MHC-II genes are induced (ON), CIITA present at the promoters interacts with CTCF bound insulators forming a second series of interactions and sub loops. While this cartoon represents the HLA-DR subregion, additional interactions are possible with these elements [48]. 